\title{
Realidad sanitaria y social de las enfermedades raras en España
}

\begin{abstract}
Karina Villar Gómez de las Heras
Médico General. Dirección General de Atención Sanitaria y Calidad. Servicio de Salud de Castilla- La Mancha. Vicepresidente de la Alianza de Familias de von Hippel-Lindau (VHL). Secretaria de la Comisión de Enfermedades Raras del Colegio Oficial de Médicos de Toledo. Miembro del Grupo de Trabajo de Genética Clínica y Enfermedades Raras de SEMFyC.
\end{abstract}

Bajo la denominación de enfermedades raras (ER) se agrupan entre cinco y siete mil patologías que presentan una gran variabilidad, tanto en sus características clínicas como en sus necesidades asistenciales e incluso sociales. Según la definición de la Unión Europea, ER son aquellas que aparecen con una frecuencia inferior a 5 por cada 10.000 habitantes. Algunas son extremadamente raras 0 ultrarraras, con pocos casos a nivel mundial (progeria, fibrodisplasia osificante progresiva), mientras que otras son mucho más frecuentes (fibrosis quística, neurofibromatosis). En conjunto se estima que alrededor de 30 millones de personas tienen una enfermedad rara en el total de los 25 países europeos.

También hay una gran diversidad en cuanto a la edad en que tienen lugar las primeras manifestaciones. El ejemplo típico de la detección (que no siempre diagnóstico) en la misma gestación o inmediatamente después del nacimiento, son los síndromes polimalformativos y las enfermedades metabólicas incluidas en las pruebas de cribado neonatal o de manifestación temprana, pero muchas otras enfermedades pasan desapercibidas en los primeros años de vida (Niemann-Pick tipo C, algunas enfermedades neuromusculares), e incluso hasta la edad adulta (von Hippel-Lindau, enfermedad de Huntington), sin que ello signifique que la enfermedad vaya a tener consecuencias menos graves para el individuo.

En el año 2005, EURORDIS (European Organization for Rare Disorders) publicó un interesante documento en el que se relacionaban una serie de características comunes a las ER':

- Son graves o muy graves, crónicas, a menudo degenerativas y ponen en peligro la vida.

- El comienzo tiene lugar en la niñez para el $50 \%$ de las ER.

- La calidad de vida de los pacientes está a veces comprometida por la falta o pérdida de autonomía, debido a las diferentes discapacidades que van apareciendo.

- Muy dolorosa en términos de carga psicosocial.
- Actualmente incurables, y por lo general sin tratamiento efectivo.

Muchas enfermedades catalogadas como raras no cumplen algunas de estas premisas. Algunas tienen tratamientos rehabilitadores, pero para otras no existen. En algunas, hay necesidades educativas especiales, en otras muchas no. Lo que sí es común a todas las ER es el desconocimiento científico y médico sobre ellas, lo cual tiene consecuencias en primer lugar para el paciente, y en segundo lugar, para su familia y su entorno. La consecuencia lógica de esto es la limitación social que ocasiona, pues si los médicos no sabemos dar respuestas, menos sabe una sociedad -ideada para gente sana- que desconoce los problemas asociados y los graves déficits de adaptación que acarrea, tanto en cuanto a barreras arquitectónicas como sociales. La consecuencia de esta falta de adaptación es que finalmente muchos de estos pacientes sufren aislamiento y/o rechazo social a todos los niveles (colegio, trabajo, acceso a hipotecas o seguros de coche, accidentes, vida, etc.).

Para favorecer el conocimiento y el estudio de las ER en España, en el año 2005 se creó mediante Orden Ministerial el "Registro de Enfermedades Raras y banco de muestras", que entró en funcionamiento en 2009. Dicho registro depende del Instituto de Salud de Enfermedades Raras, integrado a su vez en el Instituto de Salud Carlos III. Hasta la fecha, este registro tiene carácter voluntario, lo que supone un sesgo importante de información. Para tratar de solventar el problema, el Instituto está firmando acuerdos de colaboración con las Consejerías de Sanidad de diferentes Comunidades Autónomas. El portal de registro de ER (https://registroraras. isciii.es) ofrece la posibilidad de consultar patología por nombre, con enlace a la Web de Orphanet, el portal de enfermedades raras y medicamentos huérfanos (http://www.orpha.net), referencia obligada para todo investigador en este campo.

En cuanto a organismos públicos que desarrollan investigación básica en España, cabe destacar el CIBERER -Centro de Investigación Biomédica en Red de Enfermedades Raras (http://www.ciberer. es)-, del que también forma parte el Instituto de Enfermedades Raras. EI CIBERER está conforma- 
do por grupos de investigadores que desarrollan su labor en diferentes centros sanitarios de nuestra geografía. Sin embargo y como es fácil suponer, no hay grupos para todas las ER. La página Web contiene interesante información, como la localización geográfica y listado de los grupos investigadores. Ofrece la posibilidad de consultar la existencia de algún grupo investigando en una determinada patología a través de su correo electrónico gestores@ ciberer.es, y tiene un interesante apartado de documentación en el que cuelgan documentos sobre diferentes patologías ${ }^{2}$.

En cuanto a la investigación farmacológica, muchas ER no tienen, ni seguramente tendrán nunca, un tratamiento. En algunos casos el daño ya está y no habría forma de evitarlo o revertirlo (síndromes polimalformativos y con graves deficiencias intelectuales), y en otras, son patologías tan raras que a la industria farmacéutica no le es rentable investigar para encontrar un fármaco cuya venta ni siquiera va a cubrir los gastos de la investigación desarrollada. Por ello los pocos fármacos existentes se han denominado medicamentos huérfanos. Para muchas ER, la única posibilidad de que llegue a investigarse sobre un posible tratamiento existiría si dicha investigación estuviera incentivada económicamente. Además, muchos de los pocos tratamientos existentes para ER tienen un coste tan elevado que la mayoría de sistemas de salud a nivel mundial no pueden llegar a financiarlos (a la cabeza, eculizumab - Soliris $\AA$-, para la hemoglobinuria paroxística nocturna, con un coste aproximado de 300.000 euros por paciente y año). A este respecto, cabe destacar el apartado que ofrece el portal de EURORDIS en relación a los medicamentos huérfanos ${ }^{3}$.

En los últimos años, varias sociedades médicas españolas han creado en su seno grupos de trabajo integrados por médicos interesados en las ER. Cabe mencionar al grupo de Genética Clínica y Enfermedades Raras de SEMFyC, formado por médicos de familia de diferentes comunidades autónomas, algunos de ellos con algún familiar afectado por una enfermedad rara. Con el objetivo de concienciar a su colectivo, este grupo va elaborando publicaciones en relación a las ER y organiza de forma periódica un interesante taller de concienciación ${ }^{4}$, que ha presentado en diferentes congresos y jornadas médicas. Como herramienta para el médico asistencial, hace varios años pusieron en marcha el protocolo DICE-APER, el cual pretende abarcar los diferentes aspectos esenciales de la atención de estos pacientes en una consulta de Atención Primaria. Se puede consultar en la web ${ }^{5}$ y se detalla en un artículo especial publicado en este número.

En 2006 se publicó en España el Real Decreto
1302/2006 de designación y acreditación de los Centros, Servicios y Unidades de Referencia del Sistema Nacional de Salud 6 . Dicho Real Decreto no contempla la acreditación de médicos como tales, lo cual es un problema en casos concretos de ER donde no hay sino un médico interesado en la enfermedad en todo el territorio nacional. Desde 2006 hasta ahora no se ha designado ningún centro para una ER concreta. Es un proceso que los pacientes y sus familias consideran demasiado lento, pues se ven envueltos en complicados y a veces infructuosos procedimientos burocráticos de derivación entre comunidades autónomas. La crisis económica y las decisiones políticas tomadas para reducir el gasto en sanidad, han venido a agravar esta situación. Aunque este año el Ministerio de Salud ha declarado 2013 como el Año Español de las Enfermedades Raras y de nuevo se vuelve a hablar de un mapa de recursos en ER, hasta el momento los afectados no han percibido ningún cambio.

El movimiento asociativo ha desempeñado una importante labor en el conocimiento de las ER y en intentar encontrar soluciones a muchos de sus problemas. Las asociaciones de ER están formadas principalmente por los pacientes, sus familias y sus amigos, que se han organizado y buscan información, ayudados por el gran desarrollo de las tecnologías de la información que hemos experimentado en los últimos años. Así pues, muchas de estas asociaciones, formadas principalmente por voluntarios, tienen página web propia, perfil de Facebook y Twitter y se nutren de las numerosas publicaciones sobre su enfermedad que circulan por Internet. Muchas de ellas han formado federaciones que traspasan nuestras fronteras, y han empezado a organizar campañas de recaudación de fondos con los que financian sus propios proyectos de investigación, independientes de las subvenciones y ayudas públicas (Niemann-Pick, esclerosis tuberosa, telangiectasia hemorrágica hereditaria $[\mathrm{HHT}]$, Von Hippel-Lindau). Incluso han conformado grupos de discusión en diferentes idiomas, como es el caso de la plataforma RareConnect ${ }^{7}$ (impulsada por EURORDIS). También han identificado médicos que han mostrado un interés particular por su patología, y los han superespecializado, derivándoles, a través de la asociación, durante años, todos los casos que iban surgiendo. Es el caso de HHT, Wolfram, von Hippel-Lindau, Niemann Pick, mastocitosis y algunas otras, que han solicitado hace ya tiempo al Ministerio de Sanidad la acreditación de estos médicos, para salvar el problema de vivir en una comunidad autónoma diferente de la del médico experto. Sin embargo, ni aun en estos casos el Ministerio las ha acreditado todavía.

Como vemos, hay muchas iniciativas puestas en marcha en nuestro país dirigidas a mejorar el conocimiento y la atención de las ER, aunque hay que 
perfeccionarlas. En cualquier caso y desafortunadamente nunca llegarán a dar soluciones efectivas si no se consigue crear conciencia política del problema, y si no se superan las fronteras no sólo entre comunidades autónomas sino también entre naciones. Por algo el lema de EURORDIS de este año es "Traspasemos las fronteras por las enfermedades raras..., os necesitamos a todos a bordo, sobre todo, A TI".

\section{BIBLIOGRAFÍA}

1. European Organisation for Rare Diseases (EURORDIS). "Enfermedades raras: el conocimiento de esta prioridad de la Salud Pública". Diciembre, 2005. Disponible en: http://www.eurordis.org/IMG/pdf/Princeps_document-SN.pdf.

2. CIBERER. Documentación. Disponible en: http://www.ciberer.es/index.php?option=com docman\&ltemid=194

3. European Organisation for Rare Diseases (EURORDIS). "Los medicamentos huérfanos". Disponible en: http://www.eurordis.org/es/medicamentos-huérfanos.

4. Grupo de Trabajo de Genética Clínica y Enfermedades Raras de la SEMFyC. Taller interactivo "¿Pacientes raros o médicos incómodos?, las enfermedades raras en la consulta de Atención Primaria". Disponible en: http://www.gdtraras.es/
5. Sociedad Española de Medicina de Familia y Comunitaria. Protocolo DICE de Atención Primaria de Enfermedades Raras. Disponible en: http://dice-aper. semfyc.es/.

6. Real Decreto $1302 / 2006$, de 10 de noviembre, por el que se establecen las bases del procedimiento para la designación y acreditación de los Centros, Servicios y Unidades de Referencia del Sistema Nacional de Salud. Disponible en: http://www.boe.es/boe/ dias/2006/11/11/pdfs/A39503-39505.pdf

7. RareConnect. A social network of Rare Disease Communities led by NORD and EURORDIS in partnership with leading disease-specific patient groups. Disponible en: https://www.rareconnect.org.

\section{LECTURAS RECOMENDADAS}

- Enfermedades Raras: un enfoque práctico. Maravillas Izquierdo Martínez y Alfredo Avellaneda Fernández. Madrid: Instituto de Salud Carlos III, 2004. Disponible en Internet en http://bvs.isciii. es/mono/pdf/IIER_01.pdf

- Blog de enfermedades raras. http://www.webenfermedadesraras.org 